\title{
Craig Interpolation for Linear Temporal Languages *
}

\author{
Amélie Gheerbrant ${ }^{1}$ and Balder ten Cate $^{2}$ \\ 1 ILLC, Universiteit van Amsterdam, a.gheerbrant@uva.nl \\ 2 INRIA and ENS Cachan, balder.tencate@uva.nl
}

\begin{abstract}
We study Craig interpolation for fragments and extensions of propositional linear temporal logic (PLTL). We consider various fragments of PLTL obtained by restricting the set of temporal connectives and, for each of these fragments, we identify its smallest extension that has Craig interpolation. Depending on the underlying set of temporal operators, this extension turns out to be one of the following three logics: the fragment of PLTL having only the Next operator; the extension of PLTL with a fixpoint operator $\mu$ (known as linear time $\mu$-calculus); the fixpoint extension of the "Until-only" fragment of PLTL.
\end{abstract}

Key words: Propositional Linear Temporal Logic, Craig Interpolation, Linear Time $\mu$-Calculus

\section{Introduction}

Craig's interpolation theorem in classical model theory dates back from the late fifties [7]. It states that if a first-order formula $\phi$ (semantically) entails another first-order formula $\psi$, then there is an interpolant first-order formula $\theta$, such that every non-logical symbol in $\theta$ occurs both in $\phi$ and $\psi, \phi$ entails $\theta$ and $\theta$ entails $\psi$. The key idea of the Craig interpolation theorem is to relate different logical theories via their common non-logical vocabulary. In his original paper, Craig presents his work as a generalization of Beth's definability theorem, according to which implicit (semantic) definability is equivalent to explicit (syntactic) definability. Indeed, Beth's definability theorem follows from Craig's interpolation theorem, but the latter is more general.

From the point of view of applications in computer science, interpolation is often a desirable property of a logic. For instance, in fields such as automatic reasoning and software development, interpolation is related to modularization $[1,10]$, a property which allows systems or specifications to be developed efficiently by first building component subsystems (or modules). Interpolation for temporal logics is also an increasingly important topic. Temporal logics in general are widely used in systems and software verification, and interpolation has

\footnotetext{
* We are grateful to Alexandru Baltag for helpful comments and to Frank Wolter for first raising the question. The first author was supported by a GLoRiClass fellowship of the European Commission (Research Training Fellowship MEST-CT2005-020841) and the second author by the Netherlands Organization for Scientific Research (NWO) grant 639.021.508 and by ERC Advanced Grant Webdam on Foundation of Web data management.
} 


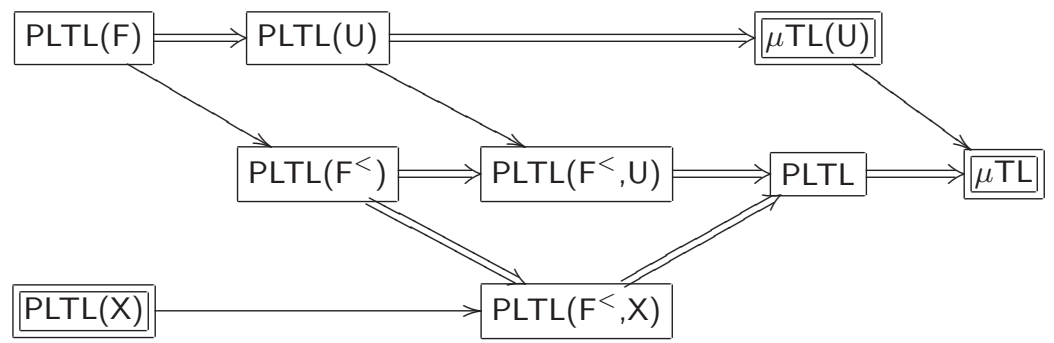

Fig. 1. Hierarchy of temporal languages

proven to be useful for building efficient model-checkers [8]. This is particularly true of a strong form of Craig interpolation known as uniform interpolation, which is quite rare in modal logic, but that the modal $\mu$-calculus satisfies (see [9]), whereas most temporal logics lack even Craig interpolation (see [17]).

We study Craig interpolation for fragments and extensions of propositional linear temporal logic (PLTL). We use the framework of [4] and work with a general notion of abstract temporal language which allows us to consider a general notion of extension of such languages. We consider different sets of temporal connectives and, for each, identify the smallest extension of the fragment of PLTL with these temporal connectives that has Craig interpolation. Depending on the set of temporal connectives, the resulting logic turns out to be either the fragment of PLTL with only the Next operator, or the extension of PLTL with a fixpoint operator $\mu$ (known as linear time $\mu$-calculus), or the fixpoint extension of the fragment of PLTL with only the Until operator (which is the stutter-invariant fragment of linear time $\mu$-calculus). The diagram in Figure 1 summarises our results. A simple arrow linking two languages means that the first one is an extension of the second one and a double arrow means that, furthermore, every extension of the first one having Craig interpolation is an extension of the second one. Temporal languages with Craig interpolation (in fact, uniform interpolation) are represented in a double frame. Thus we have for instance that $\mu \mathrm{TL}(\mathrm{U})$ is the least expressive extension of $\operatorname{PLTL}(\mathrm{F})$ with Craig interpolation.

Outline of the paper: In Section 2, we introduce a general notion of abstract temporal language. We then introduce PLTL, some of its natural fragments and its fixpoint extension known as linear time $\mu$-calculus $(\mu \mathrm{TL})$.

Section 3 contains some technical results that are used in subsequent sections. One of these relates projective definability in PLTL to definability in the fixpoint extension $\mu \mathrm{TL}$. Another result relates in a similar way $\operatorname{PLTL}(\mathrm{U})$ and $\mu \mathrm{TL}(\mathrm{U})$. Along the way, we show that $\mu \mathrm{TL}(\mathrm{U})$ is the stutter invariant fragment of $\mu \mathrm{TL}$. Stutter-invariance is a property that is argued by some authors [16] to be natural and desirable for a temporal logic. Roughly, a temporal logic is stutter-invariant if it cannot detect the addition of identical copies of a state.

In Section 4, we give three positive interpolation results. Among the fragments of PLTL obtained by restricting the set of temporal operators, we show that only one (the "Next-only" fragment) has Craig interpolation. In fact, this 
fragment satisfies a stronger form of interpolation, called uniform interpolation. The logics $\mu \mathrm{TL}$ and $\mu \mathrm{TL}(\mathrm{U})$ also have uniform interpolation.

Section 5 completes the picture by showing that $\mu \mathrm{TL}$ and $\mu \mathrm{TL}(\mathrm{U})$ are the least extensions of $\operatorname{PLTL}(F)$ and $\operatorname{PLTL}\left(F^{<}\right)$, respectively, with Craig interpolation.

\section{Preliminaries}

\subsection{Abstract Temporal Languages}

We will be dealing with a variety of temporal languages. They are all interpreted in structures consisting of a set of worlds (or, time points), a binary relation intuitively representing temporal precedence, and a valuation of proposition letters. In this section, we give an abstract model theoretic definition of temporal languages.

A flow of time, or frame, is a structure $\mathcal{T}=(W,<)$, where $W$ is a non-empty set of worlds and $<$ is a binary relation on $W$. We will focus here on $\mathbf{T}_{\omega}$, the class of linear orders of order type $\omega$, i.e., frames $(D,<)$ that are isomorphic to $(\mathbb{N},<)$, where $\mathbb{N}$ is the set of natural numbers with the natural ordering. We will also freely use $\leq$ to denote the reflexive closure of $<$.

By a propositional signature we mean a finite non-empty set of propositional letters $\sigma=\left\{p_{i} \mid i \in I\right\}$. A pointed $\sigma$-structure is a structure $\mathfrak{M}=(\mathcal{T}, V, w)$ where $\mathcal{T}=(W, R)$ is a frame, $V: \sigma \rightarrow \wp(W)$ a valuation and $w \in W$ a world. The class of all pointed $\sigma$-structures is denoted by $\operatorname{Str}[\sigma]$ and we call them $\sigma$ structures for short. Furthermore, for any class of frames $\mathbf{T}, \operatorname{Str}_{\mathbf{T}}[\sigma]$ will denote the class of $\sigma$-structures of which the underlying frame belongs to $\mathbf{T}$. Let $\sigma \subseteq \tau$ be propositional signatures. Given a $\tau$-structure $\mathfrak{M}=(\mathcal{T}, V, w)$, we define its $\sigma$-reduct $\mathfrak{M}\lceil\sigma$ as the $\sigma$-structure $(\mathcal{T}, V\lceil\sigma, w)$ where $V\lceil\sigma$ is the restriction of the valuation to the propositional letters in $\sigma$. We call $\mathfrak{M}$ a $\tau$-expansion of $\mathfrak{M}\lceil\sigma$. We also write $K\lceil\sigma$ for $\{\mathfrak{M}\lceil\sigma \mid \mathfrak{M} \in K\}$. Let $(\mathcal{T}, V, w)$ be a $\sigma$-structure and $A \subseteq W$ a subset of its domain. By $V[A / p]$, we will refer to the valuation $V$ extended with $V(p)=A$ ( $p$ being a fresh proposition letter). We will refer to the corresponding $\sigma \cup\{p\}$-expansion of $(\mathcal{T}, V, w)$ by $(\mathcal{T}, V[A / p], w)$.

Definition 1 (Abstract temporal language). An abstract temporal language (temporal language for short) is a pair $\mathcal{L}=\left(\mathcal{L}, \models_{\mathcal{L}}\right)$, where $\mathcal{L}: \sigma \mapsto \mathcal{L}[\sigma]$ is a map from propositional signatures to sets of objects that we call formulas and $=_{\mathcal{L}}$ is a relation between formulas and pointed structures satisfying the following conditions, for all propositional signatures $\sigma, \tau$ :

1. Expansion property. If $\sigma \subseteq \tau$ then $\mathcal{L}[\sigma] \subseteq \mathcal{L}[\tau]$. Furthermore, for all $\phi \in \mathcal{L}[\sigma]$ and $\mathfrak{M} \in \operatorname{Str}[\tau], \mathfrak{M} \models_{\mathcal{L}} \phi$ iff $\mathfrak{M} \uparrow \sigma \models_{\mathcal{L}} \phi$. If $\mathfrak{M} \in \operatorname{Str}[\sigma]$ and $\left.\mathfrak{M}\right|_{\mathcal{L}} \phi$, then $\phi \in \mathcal{L}[\sigma]$.

2. Closure under uniform substitution. For all $\psi \in \mathcal{L}[\sigma], p \notin \sigma$ and $\phi \in$ $\mathcal{L}[\sigma \cup\{p\}]$, there is a formula of $\mathcal{L}[\sigma]$, which we will denote by $\phi[p / \psi]$, such that for every $(\mathcal{T}, V, w) \in \operatorname{Str}[\sigma]$ the following holds:

$$
(\mathcal{T}, V, w) \models_{\mathcal{L}} \phi[p / \psi] \text { iff }\left(\mathcal{T}, V^{\prime}, w\right) \models_{\mathcal{L}} \phi
$$

where $V^{\prime}=V\left[\left\{w \mid(\mathcal{T}, V, w) \models_{\mathcal{L}} \psi\right\} / p\right]$. 
3. Negation property. For each $\phi \in \mathcal{L}[\sigma]$ there is a formula of $\mathcal{L}[\sigma]$, which we will denote by $\neg \phi$, s.t. for all $\mathfrak{M} \in \operatorname{Str}[\sigma], \mathfrak{M} \models_{\mathcal{L}} \neg \phi$ iff $\mathfrak{M} \models_{\mathcal{L}} \phi$.

For any class of frames $\mathbf{T}, \models_{\mathcal{L}, \mathbf{T}}$ will denote the restriction of $\models_{\mathcal{L}}$ to pointed structures based on $\mathbf{T}$. For $\phi \in \mathcal{L}[\sigma]$, we will use $\operatorname{Mod}^{\sigma}(\phi)$ as shorthand for $\left\{\mathfrak{M} \in \operatorname{Str}[\sigma] \mid \mathfrak{M} \models_{\mathcal{L}, \mathbf{T}} \phi\right\}$ and $\operatorname{Mod}_{\mathbf{T}}^{\sigma}(\phi)$ when restricting to a frame class $\mathbf{T}$. Whenever this is clear from the context, we will be omitting superscript and subscripts in $\operatorname{Mod}_{\mathbf{T}}^{\sigma}(\phi)$ and $\models_{\mathcal{L}, \mathbf{T}}$. We say that a class of pointed structures $\mathbf{K} \subseteq \operatorname{Str}_{\mathbf{T}}[\sigma]$ is definable in an abstract temporal language $\mathcal{L}$ (relative to the frame class $\mathbf{T})$ if there is a $\mathcal{L}$-formula $\phi$ such that for every $(\mathcal{T}, V, w) \in \operatorname{Str}_{\mathbf{T}}[\sigma]$, $(\mathcal{T}, V, w) \models \phi$ iff $(\mathcal{T}, V, w) \in \mathbf{K}$.

Definition 2 (Extension of a temporal language). Let $\mathcal{L}_{1}=\left(\mathcal{L}_{1}, \models \mathcal{L}_{1}\right)$, $\mathcal{L}_{2}=\left(\mathcal{L}_{2}, \models_{\mathcal{L}_{2}}\right)$ be temporal languages. $\mathcal{L}_{2}$ extends $\mathcal{L}_{1}$ (notation: $\left.\mathcal{L}_{1} \subseteq \mathcal{L}_{2}\right)$ if for all $\sigma$, for all $\phi \in \mathcal{L}_{1}[\sigma]$, there exists $\phi^{*} \in \mathcal{L}_{2}[\sigma]$ such that $\operatorname{Mod}(\phi)=\operatorname{Mod}\left(\phi^{*}\right)$. Also, whenever $\mathcal{L}_{1} \subseteq \mathcal{L}_{2}$, we say that $\mathcal{L}_{1}$ is a fragment of $\mathcal{L}_{2}$. Whenever restricting attention to a frame class $\mathbf{T}$ we write $\mathcal{L}_{1} \subseteq_{\mathbf{T}} \mathcal{L}_{2}$.

The following notion is related to existential second-order quantification over propositional letters. Allowing such a form of quantification in a given temporal language indeed amounts to considering its projective classes. It is a classical notion in abstract modal theory and it will be useful in the context of $\Delta$-interpolation (see Definition 15).

Definition 3 (Projective class). Let $\sigma$ be a propositional signature, $\mathbf{T}$ a frame class and let $K \subseteq \operatorname{Str}_{\mathbf{T}}[\sigma]$. Then $K$ is a projective class of a temporal language $\mathcal{L}$ relative to $\mathbf{T}$ if there is a $\phi \in \mathcal{L}[\tau]$ with $\tau \supseteq \sigma$ a propositional signature, such that $K=\operatorname{Mod}(\phi)\lceil\sigma$.

Lemma 1. Let $\mathbf{T}$ be a frame class. If $\mathcal{L}_{1} \subseteq_{\mathbf{T}} \mathcal{L}_{2}$, then every projective class of $\mathcal{L}_{1}$ relative to $\mathbf{T}$ is also a projective class of $\mathcal{L}_{2}$ relative to $\mathbf{T}$.

Definition 4 (Entailment). Let $\mathcal{L}$ be a temporal language, $\sigma$ a propositional signature, $\mathbf{T}$ a frame class and $\phi, \psi \in \mathcal{L}[\sigma]$. We say that $\phi$ entails $\psi$ in $\mathcal{L}$ over $\mathbf{T}$ and write $\phi \models_{\mathcal{L}, \mathbf{T}} \psi$ if for any $(\mathcal{T}, V, w) \in \operatorname{Str}_{\mathbf{T}}[\sigma]$, whenever $(\mathcal{T}, V, w) \models_{\mathcal{L}, \mathbf{T}} \phi$, then also $(\mathcal{T}, V, w) \models_{\mathcal{L}, \mathbf{T}} \psi$.

\subsection{Propositional Linear Temporal Logic}

Recall that $\mathbf{T}_{\omega}$ denotes the linear orders of order type $\omega$. We now introduce the syntax and semantics of PLTL, following the terminology of [11].

Definition 5 (PLTL). Let $\sigma$ be a propositional signature. The set of formulas $\operatorname{PLTL}[\sigma]$ is defined inductively, as follows:

$$
\phi, \psi:=A t|\top| \neg \phi|\phi \wedge \psi| \phi \rightarrow \psi|\phi \vee \psi| \mathrm{X} \phi|\mathrm{F} \phi| \mathrm{F}^{<} \phi \mid \phi \mathrm{U} \psi
$$

where $A t \in \sigma$. We use $\mathrm{G}$ and $\mathrm{G}^{<}$as shorthand for respectively $\neg \mathrm{F} \neg$ and $\neg \mathrm{F}^{<} \neg$. The relation $\models_{\mathrm{PLTL}}$ between PLTL-formulas and structures $(\mathcal{T}, V, w)$ is defined as follows (we only list the clauses of the temporal operators, the others are as in the case of classical propositional logic): 
- $(\mathcal{T}, V, w) \models_{\mathrm{PLTL}} \mathbf{X} \phi$ iff there exists $w^{\prime}$ such that $w<w^{\prime}$, there is no $w^{\prime \prime}$ such that $w<w^{\prime \prime}<w^{\prime}$ and $\left(\mathcal{T}, V, w^{\prime}\right) \models \phi$

- $(\mathcal{T}, V, w) \models_{\text {PLTL }} \mathbf{F} \phi$ iff there exists $w^{\prime}$ such that $w \leq w^{\prime}$ and $\left(\mathcal{T}, V, w^{\prime}\right) \models \phi$

$-(\mathcal{T}, V, w) \models_{\text {PLTL }} \mathrm{F}^{<} \phi$ iff there exists $w^{\prime}$ such that $w<w^{\prime}$ and $\left(\mathcal{T}, V, w^{\prime}\right) \models \phi$

$-\left.(\mathcal{T}, V, w)\right|_{\text {PLTL }} \phi \mathbf{U} \psi$ iff there exists $w^{\prime}$ such that $w \leq w^{\prime},\left(\mathcal{T}, V, w^{\prime}\right) \models \psi$ and for all $w^{\prime \prime}$ such that $w \leq w^{\prime \prime}<w^{\prime},\left(\mathcal{T}, V, w^{\prime \prime}\right) \models \phi$

While the above definition in principle applies to arbitrary pointed structures, the intended semantics will be, of course, in terms of structures based on frames in $\mathbf{T}_{\omega}$, and in what follows we will always restrict attention to such frames.

We define fragments $\operatorname{PLTL}(\mathcal{O})$ of PLTL by allowing in their syntax only a subset $\mathcal{O} \subseteq\left\{\mathrm{X}, \mathrm{F}^{<}, \mathrm{F}, \mathrm{U}\right\}$ of temporal operators. Note that $\mathrm{PLTL}(\mathrm{U}, \mathrm{X})$ has the same expressive power as PLTL, because $\mathrm{F} \phi$ can be defined as $\mathrm{TU} \phi$ and $\mathrm{F}^{<} \phi$ as $\mathrm{X}(\mathrm{TU} \phi)$. The same holds of $\operatorname{PLTL}\left(\mathrm{F}^{<}, \mathrm{X}\right)$ and $\operatorname{PLTL}\left(\mathrm{F}^{<}, \mathrm{X}, \mathrm{F}\right)$, as $\mathrm{F} \phi$ can be defined as $\phi \vee \mathrm{F}^{<} \phi$. Nevertheless, it is known (see [15]), that $\phi \mathbf{U} \psi$ can be defined neither in $\operatorname{PLTL}(\mathrm{F})$ nor in $\operatorname{PLTL}\left(\mathrm{F}^{<}, \mathrm{X}\right)$. Also $\mathrm{X} \phi$ and $\mathrm{F}^{<} \phi$ can be defined neither in $\operatorname{PLTL}(\mathrm{U})$ nor in $\operatorname{PLTL}(\mathrm{F})$ (we will see why later on in this paper, once we introduce the notion of stutter-invariance).

\subsection{Linear Time $\mu$-calculus}

A way of increasing the expressive power of temporal languages is to add a fixpoint operator. On arbitrary structures, adding to PLTL the least fixpoint operator $\mu$ gives the $\mu$-calculus (see for instance [9]). Here, the class of intended structures for $\mu$-calculus is restricted to those based on $\mathbf{T}_{w}$ and the resulting restricted temporal language is called $\mu \mathrm{TL}$ (see for instance [14]).

Definition $6(\mu \mathrm{TL})$. Let $\sigma$ be a propositional signature, and let $\mathcal{V}=$ $\left\{x_{1}, x_{2}, \ldots\right\}$ be a disjoint countably infinite stock of propositional variables. We define $\mu \mathrm{TL}[\sigma]$ as the set of all formulas without free variables that are generated by the following inductive definition:

$$
\phi, \psi, \xi:=A t|\top| \neg \phi|\phi \wedge \psi| \phi \rightarrow \psi|\phi \vee \psi| \mathrm{X} \phi|\mathrm{F} \phi| \mathrm{F}^{<} \phi|\phi \mathrm{U} \psi| \mu x_{i} . \xi
$$

where $A t \in \sigma \cup \mathcal{V}$ and, in the last clause, $x_{i}$ occurs only positively in $\xi$ (i.e., within the scope of an even number of negations). We will use $\nu x_{i} \cdot \phi\left(x_{i}\right)$ as shorthand for $\neg \mu x_{i} . \neg \phi\left(\neg x_{i}\right)$. The relation $\models \mu \mathrm{TL}$ is defined between $\mu \mathrm{TL}$-formulas and pointed structures $(\mathcal{T}, V, w)$ where $\mathcal{T} \in \mathbf{T}_{\omega}$. In order to define it inductively, we use an auxiliary assignment to interpret formulas with free variables. The assignment $g$ maps each free variable of $\phi$ to a set of worlds. We let $g[x \mapsto A]$ be the assignment which differ from $g$ only by assigning $A$ to $x$ and we only recall:

$-(\mathcal{T}, V, w) \models_{\mu \mathrm{PLTL}} x_{i}[g]$ iff $w \in g\left(x_{i}\right)$

- $(\mathcal{T}, V, w) \models_{\mu \mathrm{PLTL}} \mu x . \phi[g]$ iff $\forall A \subseteq W$, if $\left\{v \mid(\mathcal{T}, V, v) \models_{\mu \mathrm{TL}} \phi[g[x \mapsto A]]\right\} \subseteq$ $A$, then $w \in A$

To understand this, consider a $\mu \mathrm{TL}$-formula $\phi(x)$ and a structure $(\mathcal{T}, V, w)$ together with a valuation $g$. This formula induces an operator $F^{\phi}$ taking a set $A \subseteq W$ to the set $\left\{v:(\mathcal{T}, V, v)={ }_{\mu \mathrm{TL}} \phi(x) g[x \mapsto A]\right\} . \mu \mathrm{TL}$ is concerned 
with least fixpoints of such operators. If $\phi(x)$ is positive in $x$, the operator $F^{\phi}$ is monotone, i.e., $x \subseteq y$ implies $F^{\phi}(x) \subseteq F^{\phi}(y)$. Monotone operators $F^{\phi}$ always have a least fixpoint, defined as the intersection of all their prefixpoints: $\bigcap\{A \subseteq W:\{v:(\mathcal{T}, V, v) \models \phi(x) g[x \mapsto A]\} \subseteq A\}$ (see [3]). The formula $\mu x . \phi(x)$ denotes this least fixpoint.

It is easy to see that, for formulas without free variables, the assignment is irrelevant, and therefore $\models \mu \mathrm{TL}$ defines a binary relation between (the set of sentences of) $\mu \mathrm{TL}$ and pointed structures. In this way, $\mu \mathrm{TL}$ is an abstract modal language in the sense of Definition 1.

As before, we define a fragment $\mu \mathrm{TL}(\mathcal{O})$ for each $\mathcal{O} \subseteq\left\{\mathrm{X}, \mathrm{F}^{<}, \mathrm{F}, \mathrm{U}\right\} . \mu \mathrm{TL}(\mathrm{X})$ already as the full expressive power of $\mathrm{TL}$, since $\phi \mathrm{U} \psi$ can be defined by $\mu y .(\psi \vee$ $(\phi \wedge \mathrm{X} y)), \mathrm{F}^{<} \phi$ by $\mu y \cdot(X \phi \vee \mathrm{X} y)$ and $\mathrm{F} \phi$ by $\mu y \cdot(\phi \vee \mathrm{X} y)$. Another fragment of particular interest will be $\mu \mathrm{TL}(\mathrm{U})$. In $\mu \mathrm{TL}(\mathrm{U})$, we can still define $\mathrm{F} \phi$ in the usual way by $T \mathrm{U} \phi$, but we will see that $\mathrm{X} \phi$ and $\mathrm{F}^{<} \phi$ are not definable.

\section{Projective Definability versus Definability with Fixpoints}

In this section, we discuss two results that relate projective definability in languages without fixpoint operators to explicit definability in the corresponding language with fixpoint operators. Along the way, we also show that $\mu \mathrm{TL}(\mathrm{U})$ is the stutter-invariant fragment of $\mu \mathrm{TL}$. These results will be put to use in Section 4 and 5 .

Theorem 1. Let $\sigma$ be a propositional signature. For any $K \subseteq \operatorname{Str}_{\mathbf{T}_{\omega}}[\sigma]$, the following are equivalent:

1. $K$ is a projective class of $\operatorname{PLTL}\left(\mathrm{F}^{<}, \mathrm{X}\right)$ relative to $\mathbf{T}_{\omega}$

2. $K$ is definable by a $\mu \mathrm{TL}$ sentence relative to $\mathbf{T}_{\omega}$

Proof (Sketch). One direction follows from the fact that $\mu \mathrm{TL}$ is expressively complete for MSO on $\mathbf{T}_{\omega}$ (see [18, 3]). For the other direction, the main idea is that the existence of an accepting run of a Büchi automaton can be projectively defined by means of a PLTL-formula using only the $\mathrm{F}^{<}$and $\mathrm{X}$ operators (this is a refinement of a similar result for MSO, see [20]).

Below, we will show a similar theorem linking projective definability in $\operatorname{PLTL}(\mathrm{U})$ (which was shown in $[19,12]$ to be the stutter-invariant fragment of PLTL) to definability in $\mu \mathrm{TL}(\mathrm{U})$, which we show here to be the stutter-invariant fragment of linear time $\mu$-calculus. Before stating this second result, we first define stuttering. Intuitively, a stuttering of a linearly ordered structure $\mathfrak{M}$ is a structure obtained from $\mathfrak{M}$ by replacing each world by a non-empty finite sequence of worlds, all satisfying the same proposition letters.

Definition 7 (Stuttering). Let $\sigma$ be a propositional signature and $\mathfrak{M}=$ $((W,<), V, w), \mathfrak{M}^{\prime}=\left(\left(W^{\prime},<\right), V^{\prime}, w^{\prime}\right)$ be in $\operatorname{Str}_{\mathbf{T}_{\omega}}[\sigma]$. We say that $\mathfrak{M}^{\prime}$ is a stuttering of $\mathfrak{M}$ if and only if there is a surjective function $s: W^{\prime} \rightarrow W$ such that 
1. $s\left(w^{\prime}\right)=w$

2. for every $w_{i}, w_{j} \in W^{\prime}, w_{i}<w_{j}$ implies $s\left(w_{i}\right) \leq s\left(w_{j}\right)$

3. for every $w_{i} \in W^{\prime}$ and $p \in \sigma, w_{i} \in V^{\prime}(p)$ iff $s\left(w_{i}\right) \in V(p)$

Some notation will be useful later on. For any $w \in W$, we let $s^{-1}(w)=\left\{w^{\prime} \in\right.$ $\left.W^{\prime} \mid s\left(w^{\prime}\right)=w\right\}$. We also extend $s$ and $s^{-1}$ to subsets of $W^{\prime}$ in the following way: for any $A^{\prime} \subseteq W^{\prime}, A \in W$, we let $s\left(A^{\prime}\right)=\left\{s\left(v^{\prime}\right) \mid v^{\prime} \in A^{\prime}\right\}$ and $s^{-1}(A)=$ $\bigcup_{v \in A} s^{-1}(v)$.

Lemma 2. Let $\mathfrak{M}=((W,<), V, w), \mathfrak{M}^{\prime}=\left(\left(W^{\prime},<\right), V^{\prime}, w^{\prime}\right)$ be in $\operatorname{Str}_{\mathbf{T}_{\omega}}[\sigma]$ and $\mathfrak{M}^{\prime}$ be a stuttering of $\mathfrak{M}$, then the following hold:

1. $\forall v^{\prime} \in W^{\prime}, \forall A^{\prime} \subseteq W^{\prime}$ such that $v^{\prime} \in A^{\prime}$ implies $s^{-1}\left(s\left(v^{\prime}\right)\right) \subseteq A^{\prime}$ :

$$
\left(\left(W^{\prime},<\right), V^{\prime}\left[A^{\prime} / p\right], v^{\prime}\right) \text { is a stuttering of }\left((W,<), V\left[s\left(A^{\prime}\right) / p\right], s\left(v^{\prime}\right)\right)
$$

2. $\forall v \in W, \forall A \subseteq W, \forall v^{\prime} \in s^{-1}(v)$ :

$$
\left(\left(W^{\prime},<\right), V^{\prime}\left[s^{-1}(A) / p\right], v^{\prime}\right) \text { is a stuttering of }((W,<), V[A / p], v)
$$

Definition 8 (Stutter-Invariant Class of Pointed Structures). Let $\sigma$ be a propositional signature and $\mathbf{K} \subseteq \operatorname{Str}_{\mathbf{T}_{\omega}}[\sigma]$. Then $\mathbf{K}$ is a stutter-invariant class relative to $\mathbf{T}_{\omega}$ iff for every $\mathfrak{M} \subseteq \operatorname{Str}_{\mathbf{T}_{\omega}}[\sigma]$ and for every stuttering $\mathfrak{M}^{\prime}$ of $\mathfrak{M}$, $\mathfrak{M} \in \mathbf{K} \Leftrightarrow \mathfrak{M}^{\prime} \in \mathbf{K}$.

Definition 9 (Stutter-free Pointed Structure). We say that a pointed structure $\mathfrak{M}$ is stutter-free whenever for all $\mathfrak{M}^{\prime}$ such that $\mathfrak{M}$ is a stuttering of $\mathfrak{M}^{\prime}, \mathfrak{M}^{\prime}$ is isomorphic to $\mathfrak{M}$.

Only stutter-invariant classes of structures in $\operatorname{Str}_{\mathbf{T}_{\omega}}[\sigma]$ are definable in $\operatorname{PLTL}(\mathrm{U})$ and $\mu \mathrm{TL}(\mathrm{U})$. This is known for $\operatorname{PLTL}(\mathrm{U})$ (see $[12,19]$ ), but it also holds for $\mu \mathrm{TL}(\mathrm{U})$.

Proposition 1. Let $\sigma$ be a propositional signature. For every $\mu \mathrm{TL}(\mathrm{U})$-sentence $\phi$ in signature $\sigma, \operatorname{Mod}(\phi)$ is stutter-invariant.

Proof. By induction on the sentence complexity. For the sake of the induction, we can use expanded $\sigma$-structures as in classical model theory. Hence we consider two base cases, one for propositional letters and one for propositional variables. The propositional letter case is clear. We handle the propositional variable case $x_{i}$ similarly, except that we use $\sigma$-models expanded with the value of $x_{i}$ (i.e., models considered together with a partial auxiliary valuation, so that $x_{i}$ can be seen as a sentence). The induction hypothesis says that for any propositional signature $\sigma$ and $\mu \mathrm{TL}(\mathrm{U})$-sentence $\phi$ of complexity $n$ in signature $\sigma, \operatorname{Mod}(\phi)$ is a stutter-invariant invariant class. Now consider the case were $\phi$ is of complexity $n+1$. We handle the Boolean connectives and the $\mathrm{U}$ operator as in the PLTL(U) case. Now suppose $\phi: \approx \mu x \cdot \psi(x)$. We want to show that for every $\mathfrak{M} \subseteq \operatorname{Str}_{\mathbf{T}}[\sigma]$ and for every stuttering $\mathfrak{M}^{\prime}$ of $\mathfrak{M}$ :

$\mathfrak{M}=((<, W), V, w) \in \operatorname{Mod}(\mu x . \psi(x)) \Leftrightarrow \mathfrak{M}^{\prime}=\left(\left(<, W^{\prime}\right), V^{\prime}, w^{\prime}\right) \in \operatorname{Mod}(\mu x . \psi(x))$ 
For the left to right direction, suppose $((W,<), V, w) \models \mu x . \psi(x)$, i.e., $\forall A \subseteq W$, if $\{v \mid((W,<), V[A / p], v)=\psi(p)\} \subseteq A$, then $w \in A$. Consider $A^{\prime} \subseteq W^{\prime}$ such that $\left\{v \mid\left(\left(W^{\prime},<\right), V^{\prime}\left[A^{\prime} / p\right], v\right) \models \psi(p)\right\} \subseteq A^{\prime}$. We want to show that $w^{\prime} \in A^{\prime}$. Let us first show that $v^{\prime} \in A^{\prime}$ implies $s^{-1}\left(s\left(v^{\prime}\right)\right) \subseteq A^{\prime}$. For every $v^{\prime} \in A^{\prime}$, we have that $\left(\left(W^{\prime},<\right), V^{\prime}\left[A^{\prime} / p\right], v^{\prime}\right) \models \psi(p)$. Now by induction hypothesis for any $v \in s^{-1}\left(s\left(v^{\prime}\right)\right),\left(\left(W^{\prime},<\right), V^{\prime}\left[A^{\prime} / p\right], v\right) \models \psi(p)$ and by hypothesis on $A^{\prime}$, $v \in A^{\prime}$. It follows from this property of $A^{\prime}$ that $\mathfrak{M}^{\prime}$ being a stuttering of $\mathfrak{M}$, by Lemma 2 for any $v^{\prime} \in W^{\prime},\left(\left(<, W^{\prime}\right), V^{\prime}\left[A^{\prime} / p\right], v^{\prime}\right)$ is also a stuttering of $\left((<, W), V\left[s\left(A^{\prime}\right) / p\right], s\left(v^{\prime}\right)\right)$ and by induction hypothesis:

$$
\left(\left(W^{\prime},<\right), V^{\prime}\left[A^{\prime} / p\right], v^{\prime}\right) \models \psi(p) \text { iff }\left((<, W), V\left[s\left(A^{\prime}\right) / p\right], s\left(v^{\prime}\right)\right) \models \psi(p)
$$

Hence $\left\{v \mid\left((W,<), V\left[s\left(A^{\prime}\right) / p\right], v\right) \models \psi(p)\right\} \subseteq s\left(A^{\prime}\right)$. But $\mathfrak{M} \models \mu x . \psi(x)$. It follows that $w \in S\left(A^{\prime}\right)$, so $s(w) \in A^{\prime}$, i.e., $w^{\prime} \in A^{\prime}$.

Now for the right to left direction, suppose $\left(\left(W^{\prime},<\right), V^{\prime}, w^{\prime}\right) \models \mu x . \psi(x)$, i.e., $\forall A^{\prime} \subseteq W^{\prime}$, if $\left\{v \mid\left(W^{\prime},<\right), V^{\prime}\left[A^{\prime} / p\right], v \models \psi(p)\right\} \subseteq A^{\prime}$, then $w^{\prime} \in A^{\prime}$. Consider $A \subseteq W$ such that $\{v \mid(W,<), V[A / p], v \models \psi(p)\} \subseteq A$. We want to show that $w \in A$. $\mathfrak{M}^{\prime}$ being a stuttering of $\mathfrak{M}$, by Lemma 2, for any $v \in W, v^{\prime} \in s^{-1}(v)$, $\left(\left(<, W^{\prime}\right), V^{\prime}\left[s^{-1}(A) / p\right], v^{\prime}\right)$ is also a stuttering of $((<, W), V[A / p], v)$ and by induction hypothesis, for any $v \in W, v^{\prime} \in s^{-1}(v)$ :

$$
\left(\left(W^{\prime},<\right), V^{\prime}\left[s^{-1}(A) / p\right], v^{\prime}\right) \models \psi(p) \text { iff }((W,<), V[A / p], v) \models \psi(p)
$$

Hence $\left\{v \mid\left(\left(W^{\prime},<\right), V^{\prime}\left[s^{-1}(A) / p\right], v\right) \models \psi(p)\right\} \subseteq s^{-1}(A)$. But $\mathfrak{M}^{\prime} \models \mu x . \psi(x)$. It follows that $w^{\prime} \in s^{-1}(A)$, so $s^{-1}\left(w^{\prime}\right) \subseteq A$, i.e., $w \in A$.

Corollary 1. Let $K \subseteq S t r_{\mathbf{T}_{\omega}}[\sigma]$ be stutter-invariant and let $\phi \in \mu \mathrm{TL}(\mathrm{U})[\sigma]$ be a sentence such that for each stutter-free $\mathfrak{M} \in \operatorname{Str}_{\mathbf{T}_{\omega}}[\sigma], \mathfrak{M} \models \phi$ if and only if $\mathfrak{M} \in K$. Then $\phi$ defines $K$.

We now show that (over $\mathbf{T}_{\omega}$ ) $\mu \mathrm{TL}(\mathrm{U})$ is the stutter-invariant fragment of $\mu T L$. The proof is a variant of [19], where Peled and Wilke show that stutterinvariant PLTL properties are expressible without $X$. We give it in detail, as the construction procedure below will be useful again later on in the paper.

Lemma 3. Let $\sigma$ be a modal vocabulary. For every $\mu \mathrm{TL}$ sentence $\phi$ in vocabulary $\sigma$, there exists a $\mu \mathrm{TL}(\mathrm{U})$ sentence $\phi^{*}$ in vocabulary $\sigma$ that agrees with $\phi$ on all stutter-free structures over $\mathbf{T}_{\omega}$ :

$$
\mathfrak{M}=\phi \leftrightarrow \phi^{*} \text { for all stutter free pointed structures } \mathfrak{M} \in \operatorname{Str}_{\mathbf{T}_{\omega}}[\sigma]
$$

Proof. Assume $\sigma=\left\{p_{0}, \ldots, p_{n-1}\right\}$. The proof goes by induction on the structure of $\phi$. For convenience, we use expanded structures. The base case is clear: $p^{*}=p$ for any propositional variable or letter $p$. Now as regards the induction step, we can set $(\neg \psi)^{*}=\neg \psi^{*},(\psi \wedge \xi)^{*}=\psi^{*} \wedge \tau^{*},(\psi \mathrm{U} \xi)^{*}=\psi^{*} \mathrm{U} \xi^{*},(\mu x . \psi)^{*}=\mu x . \psi^{*}$. If $\phi$ is of the form $\mathrm{X} \psi$, we let $B$ be the set of all possible valuations $\sigma \rightarrow\{\perp, \top\}$, and for each $g \in B$, we let $\beta_{g}$ be the formula $\alpha_{0} \wedge \ldots \wedge \alpha_{n-1}$ where $\alpha_{j}=p_{j}$ if $g\left(p_{j}\right)=\top$ and $\alpha_{j}=\neg p_{j}$ if $g\left(p_{j}\right)=\perp$. Now observe that if $g, g^{\prime} \in B$ are such that $g \neq g^{\prime}$, then 


$$
\mathfrak{M}, w \models \beta_{g} \wedge \mathrm{X} \beta_{g^{\prime}} \leftrightarrow \beta_{g} \cup \beta_{g^{\prime}} \text { for } \mathfrak{M} \in \operatorname{Str}_{\mathbf{T}}[\sigma] \text { stutter-free }
$$

We have $\mathfrak{M}, w \models \mathbf{X} \psi$ if and only if every point in it satisfies the same set of proposition letters and $\mathfrak{M}, w \models \psi$, or the valuation function doesn't send the same set of proposition letters to $w$ and to its immediate successor $w^{\prime}$ and $\mathfrak{M}, w^{\prime}=\phi$. Thus we can set:

$$
(\mathrm{X} \psi)^{*}=\bigvee_{g \in G}\left(\left(\mathrm{G} \beta_{g} \wedge \psi^{*}\right) \vee \bigvee_{g \neq g^{\prime}}\left(\beta_{g} \mathrm{U}\left(\beta_{g^{\prime}} \wedge \psi^{*}\right)\right)\right)
$$

Theorem 2. Let $\phi \in \mu \mathrm{TL}[\sigma]$ be a sentence such that $\operatorname{Mod}(\phi)$ is stutterinvariant. Then there exists $\phi^{*} \in \mu \mathrm{TL}(\mathrm{U})[\sigma]$ such that $\operatorname{Mod}(\phi)=\operatorname{Mod}\left(\phi^{*}\right)$.

Proof. Follows from Lemma 3 and Corollary 1.

Following [12], we now introduce a variant of the notion of projective class, that we call harmonious projective class, which preserves stutter-invariance. Before we define it, we first introduce the notion of a harmonious expansion. For any propositional signature $\sigma$ and worlds $w, w^{\prime}$, we write $w \equiv_{\sigma} w^{\prime}$ if $w$ and $w^{\prime}$ satisfy the same propositions in $\sigma$.

Definition 10 (Harmonious expansion). Let $\sigma \subseteq \tau$ be propositional signatures and $\mathfrak{M} \in \operatorname{Str}_{\mathbf{T}_{\omega}}[\tau]$. We say that $\mathfrak{M}$ is a harmonious expansion of $\mathfrak{M} \uparrow \sigma$ whenever $\forall w, w^{\prime} \in W$ such that $w^{\prime}$ is a direct successor of $w, w \equiv_{\sigma} w^{\prime}$ implies $w \equiv_{\tau} w^{\prime}$.

Definition 11 (Harmonious projective class). Let $\sigma$ be a propositional signature and $K \subseteq \operatorname{Str}_{\mathbf{T}_{\omega}}[\sigma]$. Then $K$ is a harmonious projective class of a temporal language $\mathcal{L}$ relative to $\mathbf{T}_{\omega}$ whenever there is $\phi \in \mathcal{L}[\tau]$ with $\tau \supseteq \sigma$ such that for all $\mathfrak{M} \in \operatorname{Str}_{\mathbf{T}_{\omega}}[\sigma]: \mathfrak{M} \in K$ iff there is a harmonious $\tau$-expansion $\mathfrak{M}^{+}$of $\mathfrak{M}$ such that $\mathfrak{M}^{+} \models \phi$.

We will be using the following proposition in order to show Theorem 3. It refers to the notion of $\omega$-regular language, cf. [20]. We do not define this notion here as it is not central in this paper. The proof of the proposition in [12] uses a notion of stutter-invariant automata.

Proposition 2 ([12]). On $\mathbf{T}_{\omega}$, harmonious projective classes of $\mathrm{PLTL}(\mathrm{U})$ define exactly the stutter-invariant $\omega$-regular languages.

Now we are able to show the following theorem:

Theorem 3. Let $\sigma$ be a propositional signature. For any $K \subseteq \operatorname{Str}_{\mathbf{T}}[\sigma]$, the following are equivalent:

1. $K$ is a harmonious projective class of $\mathrm{PLTL}(\mathrm{U})$ relative to $\mathbf{T}_{\omega}$

2. $K$ is definable by a $\mu \mathrm{TL}(\mathrm{U})$-sentence $\phi$ relative to $\mathbf{T}_{\omega}$

Proof. Follows from Theorem 1 and Proposition 2, because by [12, 19], PLTL(U) is the stutter-invariant fragment of PLTL and by Theorem 2, $\mu \mathrm{TL}(\mathrm{U})$ is the stutter-invariant fragment of $\mu \mathrm{TL}$. 


\section{Temporal Languages with Craig Interpolation}

In this section, we show that three of the temporal languages previously discussed have Craig interpolation.

Definition 12 (Craig interpolation property). Let $\mathcal{L}$ be a temporal language and $\mathbf{T}$ a frame class. Then $\mathcal{L}$ has the Craig interpolation property over $\mathbf{T}$ whenever the following holds. Let $\phi \in \mathcal{L}[\sigma], \psi \in \mathcal{L}\left[\sigma^{\prime}\right]$. Whenever $\phi=_{\mathcal{L}, \mathbf{T}} \psi$, then there exists $\theta \in \mathcal{L}\left[\sigma \cap \sigma^{\prime}\right]$ such that $\phi \models_{\mathcal{L}, \mathbf{T}} \theta$ and $\theta \models_{\mathcal{L}, \mathbf{T}} \psi$.

They even satisfy a stronger form of interpolation, which is called uniform interpolation. Intuitively, if a temporal language has uniform interpolation, it means that the interpolant can be constructed so that it depends only on the signature of the antecedent and its intersection with the signature of the consequent.

Definition 13 (Uniform Interpolation). Let $\mathcal{L}$ be a temporal language and $\mathbf{T}$ a frame class. $\mathcal{L}$ has the uniform interpolation property over $\mathbf{T}$ if, for all signatures $\sigma \subseteq \tau$ and for each formula $\phi \in \mathcal{L}[\tau]$ there is a formula $\theta \in \mathcal{L}[\sigma]$ such that $\phi \models_{\mathcal{L}} \theta$ and for each formula $\psi \in \mathcal{L}\left[\tau^{\prime}\right]$ with $\tau \cap \tau^{\prime} \subseteq \sigma$, if $\phi \models_{\mathcal{L}} \psi$ then $\theta \mid{ }_{\mathcal{L}} \psi$.

Theorem 4. $\mu \mathrm{TL}$ has uniform interpolation over $\mathbf{T}_{\omega}$.

Proof. MSO has uniform interpolation (for monadic predicates) on any class of structures (so in particular on $\mathbf{T}_{\omega}$ ) because it has set quantifiers (see [8]). By $[18,3], \mu \mathrm{TL}$ is expressively complete for MSO. Hence $\mu \mathrm{TL}$ uniform interpolants can always be obtained via translation into MSO.

Theorem 5. $\mu \mathrm{TL}(\mathrm{U})$ has uniform interpolation over $\mathbf{T}_{\omega}$.

Proof. Let $\sigma \subseteq \tau$ be modal signatures and let $\phi \in \mu \mathrm{TL}(\mathrm{U})[\tau]$. By Theorem 4, there exists $\theta \in \mu \mathrm{TL}[\sigma]$ such that $\phi=\theta$ and for each formula $\psi \in \mu \mathrm{TL}\left[\tau^{\prime}\right]$ with $\tau \cap \tau^{\prime} \subseteq \sigma$, if $\phi \models \psi$, then $\theta \models \psi$. Now let $\theta^{*} \in \mu \mathrm{TL}(\mathrm{U})$ be the formula that agrees with $\theta$ on all stutter-free structures based on $\mathbf{T}_{\omega}$ (by Lemma 3, such a formula exists). We want to show that $\phi=\theta^{*}$ and that for each formula $\psi \in \mu \operatorname{TL}(\mathrm{U})\left[\tau^{\prime}\right]$ with $\tau \cap \tau^{\prime} \subseteq \sigma$, if $\phi \models \psi$, then $\theta^{*} \models \psi$. Let $\operatorname{SMod}(\phi)$ denote the set of stutter free structures in $\operatorname{Mod}(\phi)$. As $\operatorname{Mod}(\phi) \subseteq \operatorname{Mod}(\theta), \operatorname{SMod}(\phi) \subseteq \operatorname{SMod}(\theta)$. Now by construction of $\theta^{*}$ also $\operatorname{SMod}(\phi) \subseteq \operatorname{SMod}\left(\theta^{*}\right) . \operatorname{Mod}(\phi)$ and $\operatorname{Mod}\left(\theta^{*}\right)$ are both stutter-invariant classes. It follows from Corollary 1 that the closure under stuttering of $\operatorname{SMod}(\phi)$ is included in the closure under stuttering of $\operatorname{SMod}\left(\theta^{*}\right)$, i.e., $\operatorname{Mod}(\phi) \subseteq \operatorname{Mod}\left(\theta^{*}\right)$, i.e., $\phi=\theta^{*}$. The argument for $\theta^{*}=\psi$ is similar.

Theorem 6. PLTL $(\mathrm{X})$ has uniform interpolation over $\mathbf{T}_{\omega}$.

Proof (Sketch). We will show something much stronger, namely that every projective class of $\operatorname{PLTL}(X)$ is definable by a $\operatorname{PLTL}(X)$-formula.

Let $\phi \in \operatorname{PLTL}(\mathrm{X})[\sigma \cup\{p\}]$. We will show how to construct a formula $\psi \in \operatorname{PLTL}(\mathrm{X})[\sigma]$ that defines the class of $\sigma$-reducts of models of $\phi$. Let $n$ be the maximal nesting depth of $\mathrm{X}$-operators in $\phi$. Intuitively, $\phi$ can only talk about 
the first $n$ world in the pointed structure (starting from the designated world). We can represent every valuation of $p$ in these $n$ worlds by a set $S \subseteq\{0, \ldots, n\}$, where $k \in S$ represents that $p$ is true at the $k$-th world starting from the designated world. For each $S \subseteq\{1, \ldots, n\}$ we define $\phi^{S}$ as follows: we replace each occurrence of $p$ in $\phi$ that is in the scope $k$ X-operators $(k \leq n)$ by $\top$ if $k \in S$ and $\perp$ otherwise. Then $\phi$ and $\phi^{S}$ are equivalent in all pointed structures in which the valuation of $p$ is as described by $S$. This can be shown by a formula induction. Now, let $\psi=\bigvee_{S \subseteq\{0, \ldots, n\}} \phi^{S}$. Then $\psi$ holds in a pointed $\sigma$-structure $\mathfrak{M}$ iff $\mathfrak{M}$ has an expansion satisfying $\phi$.

\section{Interpolation Closure Results for Temporal Languages}

In this section, we look at the fragments of PLTL that do not have Craig interpolation, and we address the question how much expressive power must be added in order to regain interpolation. We will phrase our main results in terms of the notion of interpolation closure, which we define by taking inspiration from abstract model theory (see [4]):

Definition 14 (Interpolation Closure). Let $\mathbf{T}$ be a frame class. $\mathcal{L}_{2}$ is the interpolation closure of $\mathcal{L}_{1}$ over $\mathbf{T}$ if $\mathcal{L}_{1} \subseteq_{\mathbf{T}} \mathcal{L}_{2}, \mathcal{L}_{2}$ has interpolation over $\mathbf{T}$, and for every abstract temporal language $\mathcal{L}_{3}$, if $\mathcal{L}_{1} \subseteq \mathcal{L}_{3}$ and $\mathcal{L}_{3}$ has Craig interpolation on $\mathbf{T}$, then $\mathcal{L}_{2} \subseteq_{\mathbf{T}} \mathcal{L}_{3}$.

\subsection{The Interpolation Closure of $\operatorname{PLTL}\left(\mathrm{F}^{<}\right)$}

A useful tool for proving interpolation closure results is the following lemma (see [4]):

Definition 15 ( $\Delta$-interpolation property). Let $\mathcal{L}$ be a temporal language and $\mathbf{T}$ a frame class. Then $\mathcal{L}$ has the $\Delta$-interpolation property over $\mathbf{T}$ whenever the following holds: let $\sigma$ be a propositional signature and $K \subseteq \operatorname{Str}_{\mathbf{T}}[\sigma]$, if both $K$ and $\bar{K}$ are projective classes of $\mathcal{L}$ relative to $\mathbf{T}$, there is a $\mathcal{L}$-formula $\phi$ such that $K=\operatorname{Mod}_{\mathbf{T}}^{\sigma}(\phi)$.

Lemma 4. Let $\mathcal{L}$ be a temporal language with Craig interpolation on $\mathbf{T}_{\omega}$. Then $\mathcal{L}$ has $\Delta$-interpolation over $\mathbf{T}_{\omega}$.

The proof of Lemma 4 is similar to the one given in [6] (we only need to remark that the substitution property assumed here of abstract temporal languages is stronger thanimplies the renaming property assumed in [6] of abstract modal languages).

Now we will show that $\operatorname{PLTL}\left(\mathrm{F}^{<}, \mathrm{X}\right)$ is contained in the interpolation closure of $\operatorname{PLTL}\left(\mathrm{F}^{<}\right)$over $\mathbf{T}_{\omega}$. As an intermediate step, we show that in every extension of $\operatorname{PLTL}\left(\mathrm{F}^{<}\right)$having Craig interpolation, the property $\mathrm{X} p$ is "definable". By this, we mean the following:

Lemma 5. Let $\mathcal{L}$ be an extension of $\operatorname{PLTL}\left(\mathrm{F}^{<}\right)$with Craig-interpolation over $\mathbf{T}_{\omega}$. Then there is $\xi \in \mathcal{L}[\{p\}]$ such that $\operatorname{Mod}(\xi)=\operatorname{Mod}\left(\mathrm{X}_{p}\right)$. 
Proof. Let $q, r$ be new distinct propositional letters. Consider the two following projective classes of $\operatorname{PLTL}\left(\mathrm{F}^{<}\right): \operatorname{Mod}\left(\mathrm{F}^{<}(p \wedge q) \wedge \neg \mathrm{F}^{<} \mathrm{F}^{<} q\right) \uparrow\{p\}$ and $\operatorname{Mod}\left(\left(\mathrm{F}^{<}(\neg p \wedge r) \wedge \neg \mathrm{F}^{<} \mathrm{F}^{<} r\right) \vee \mathrm{G}^{<} \perp\right) \uparrow\{p\}$. As $\operatorname{PLTL}\left(\mathrm{F}^{<}\right) \subseteq \mathcal{L}$, these two classes are also projective classes of $\mathcal{L}$ (by Lemma 1). They also complement each other, as a $\{p\}$-structure belongs to the first class exactly when the first node of this structure has a successor node where $p$ holds and it belongs to the second class in all other cases. By $\Delta$-interpolation for $\mathcal{L}$ on $\mathbf{T}$, it follows that the first class is definable in $\mathcal{L}$ by means of some formula $\xi$ in signature $\{p\}$, i.e., there is $\xi \in \mathcal{L}[\{p\}]$ such that $\operatorname{Mod}(\mathbf{X} p)=\operatorname{Mod}(\xi)$.

Theorem 7. Every extension of $\operatorname{PLTL}\left(\mathrm{F}^{<}\right)$with Craig interpolation over $\mathbf{T}_{\omega}$ is an extension of $\operatorname{PLTL}\left(\mathrm{F}^{<}, \mathrm{X}\right)$ over $\mathbf{T}_{\omega}$.

Proof. Let $\mathcal{L}$ be an extension of $\operatorname{PLTL}\left(\mathrm{F}^{<}\right)$with Craig interpolation over $\mathbf{T}_{\omega}$ and $\sigma$ a propositional signature. We show by induction on the complexity of $\phi$ (number of Boolean and temporal operators in $\phi$ ) that for all $\phi \in \operatorname{PLTL}\left(\mathrm{F}^{<}, \mathrm{X}\right)[\sigma]$, there exists $\phi^{\prime} \in \mathcal{L}[\sigma]$ such that $\operatorname{Mod}(\phi)=\operatorname{Mod}\left(\phi^{\prime}\right)$. The base case is clear. The induction hypothesis says that for all $\sigma$, for all $\phi \in \operatorname{PLTL}\left(\mathrm{F}^{<}, \mathrm{X}\right)[\sigma]$ of complexity at most $n$, there exists $\phi^{\prime} \in \mathcal{L}[\sigma]$ such that $\operatorname{Mod}(\phi)=\operatorname{Mod}\left(\phi^{\prime}\right)$. Now let $\phi$ be of complexity $n+1$. If $\phi:=\mathbf{X} \psi$, by induction hypothesis there exists $\psi^{\prime} \in \mathcal{L}[\sigma]$ such that $\operatorname{Mod}(\psi)=\operatorname{Mod}\left(\psi^{\prime}\right)$. Pick any $p \notin \sigma$. By Lemma 5 and the expansion property we know:

1. There is $\xi \in \mathcal{L}[\sigma \cup\{p\}]$ such that $\operatorname{Mod}(\mathrm{X} p)=\operatorname{Mod}(\xi)$.

We will define $\phi^{\prime}$ as $\xi\left[p / \psi^{\prime}\right] \in \mathcal{L}[\sigma]$ (by closure under uniform substitution of $\mathcal{L}$, such a formula exists). We need to show that $\operatorname{Mod}(\mathbf{X} \psi)=\operatorname{Mod}\left(\xi\left[p / \psi^{\prime}\right]\right)$. From 1 we can derive as a particular case:

2. For any $(\mathcal{T}, V, w) \in \operatorname{Str}_{\mathbf{T}}[\sigma \cup\{p\}]$ where $V(p)=\left\{w_{i} \mid\left(F, V, w_{i}\right) \models \psi^{\prime}\right\}$, $(\mathcal{T}, V, w) \models \xi$ iff there exists $w^{\prime} \in D$ such that $w<w^{\prime}$, there is no $w^{\prime \prime}$ such that $w<w^{\prime \prime}<w^{\prime}$ and $\left(\mathcal{T}, V, w^{\prime}\right)=p$.

Now by closure under uniform substitution of $\mathcal{L}, 2$ is equivalent to the following:

3. For any $(\mathcal{T}, V, w) \in \operatorname{Str}_{\mathbf{T}}[\sigma],(F, V, w) \models \xi\left[p / \psi^{\prime}\right]$ iff there exists $w^{\prime} \in D$ such that $w<w^{\prime}$, there is no $w^{\prime \prime}$ such that $w<w^{\prime \prime}<w^{\prime}$ and $\left(F, V, w^{\prime}\right)=p\left[p / \psi^{\prime}\right]$.

Finally, $\psi^{\prime}$ and $p\left[p / \psi^{\prime}\right]$ holding exactly in the same models, we can replace $p\left[p / \psi^{\prime}\right]$ by $\psi^{\prime}$ in the second member of the equivalence in 3 . Hence $\operatorname{Mod}(\mathbf{X} \psi)=$ $\operatorname{Mod}\left(\xi\left[p / \psi^{\prime}\right]\right)$. We can use similar arguments for the operator $\mathbf{F}^{<}$and for Boolean connectives.

By putting Lemma 4 to use, we now improve Theorem 7 and identify the interpolation closure of $\operatorname{PLTL}\left(\mathrm{F}^{<}\right)$.

Theorem 8. $\mu \mathrm{TL}$ is the interpolation closure of $\operatorname{PLTL}\left(\mathrm{F}^{<}, \mathrm{X}\right)$ over $\mathbf{T}_{\omega}$.

Proof. Let $\sigma$ be a propositional signature. Now let $K \subseteq \operatorname{Str}_{\mathbf{T}_{\omega}}[\sigma]$ be definable by a $\mu \mathrm{TL}$-sentence $\phi$ in signature $\sigma$. As $\mu \mathrm{TL}$ is closed under negation, there is a $\mu \mathrm{TL}$ sentence $\neg \phi$ in signature $\sigma$, which defines the complement of $K$ over $\operatorname{Str}_{\mathbf{T}_{\omega}}[\sigma]$. 
It follows by Theorem 1 that both $K$ and its complement are projective classes of $\operatorname{PLTL}\left(\mathrm{F}^{<}, \mathrm{X}\right)$. Now consider a temporal language $\mathcal{L} \supseteq \operatorname{PLTL}\left(\mathrm{F}^{<}, \mathrm{X}\right)$ with Craig interpolation over $\mathbf{T}_{\omega}$. By Lemma $1, K$ and its complement are also projective classes of $\mathcal{L}$ and by Lemma 4 , it follows that $K$ is definable in $\mathcal{L}$.

\subsection{The Interpolation Closure of PLTL(F)}

For the case of the stutter-invariant languages PLTL(F) and PLTL(U), we need to refine the notion of $\Delta$-interpolation, by considering harmonious projective classes.

Definition 16 (Harmonious $\Delta$-interpolation property). Let $\mathcal{L}$ be a temporal language. Then $\mathcal{L}$ has the harmonious $\Delta$-interpolation property over $\mathbf{T}_{\omega}$ whenever the following holds. Let $K$ be a class of $\mathcal{L}$-structures based on $\mathbf{T}_{\omega}$. If both $K$ and $\bar{K}$ are harmonious projective classes of $\mathcal{L}$ relative to $\mathbf{T}_{\omega}$, there is a $\mathcal{L}$-formula $\phi$ such that $K=\operatorname{Mod}_{\mathbf{T}_{\omega}}(\phi)$.

Lemma 6. If $\mathcal{L}_{1} \subseteq \mathcal{L}_{2}$, then every harmonious projective class of $\mathcal{L}_{1}$ is also a harmonious projective class of $\mathcal{L}_{2}$.

Definition 17 (Harmonious temporal language). A temporal language $\mathcal{L}$ is harmonious for $\mathbf{T}_{\omega}$ if the following holds. For every $\sigma \subseteq \tau$ propositional signatures, there is a formula $\phi \in \mathcal{L}[\tau]$ such that for every $\mathfrak{M} \in \operatorname{Str}_{\mathbf{T}_{\omega}}[\tau], \mathfrak{M}=\phi$ if and only if $\mathfrak{M}$ is an harmonious expansion of $\mathfrak{M}\lceil\sigma$.

Proposition 3. PLTL(U) and its extensions are harmonious for $\mathbf{T}_{\omega}$.

Proof. Fix $\sigma \subseteq \tau$ with $|\sigma|=n,|\tau \backslash \sigma|=m$. We can represent any valuation over $\sigma$ by a finite conjunction of atoms and negations of atoms. Let $\left\{\sigma_{i} \mid i \in 2^{n}\right\}$ be the set of all such conjunctions. Also, for each $\sigma_{i}$, we define the corresponding set $\left\{\tau_{j}^{i} \mid j \in 2^{m}\right\}$ as the set of conjunctions representing all possible ways of extending to $\tau$ the valuation represented by $\sigma_{i}$. Now for every $\mathfrak{M} \in \operatorname{Str}_{\mathbf{T}}[\tau]$,

$$
\mathfrak{M} \models \bigwedge_{i, j \in 2^{n}}\left(\sigma_{i} \mathrm{U} \sigma_{j} \rightarrow \bigvee_{k, l \in 2^{m}} \tau_{k}^{i} \mathrm{U} \tau_{l}^{j}\right)
$$

if and and only if $\mathfrak{M}$ is an harmonious expansion of $\mathfrak{M} \uparrow \sigma$, i.e., $\operatorname{PLTL}(U)$ is harmonious. It is immediate from definition 2 that every extension of a temporal language which is harmonious for $\mathbf{T}_{\omega}$ is also harmonious for $\mathbf{T}_{\omega}$.

Lemma 7. Let $\mathcal{L}$ be a temporal language which has Craig interpolation and is harmonious for $\mathbf{T}_{\omega}$. Then $\mathcal{L}$ has harmonious $\Delta$-interpolation over $\mathbf{T}_{\omega}$.

$\mathcal{L}$ being harmonious, we can use the formula $\phi$ in Definition 17 and appeal for the proof of Lemma 7 to the same classical argument as for Lemma 4.

Theorem 9. Every extension of $\mathrm{PLTL}(\mathrm{F})$ with Craig interpolation over $\mathbf{T}_{\omega}$ is an extension of $\mathrm{PLTL}(\mathrm{U})$ over $\mathbf{T}_{\omega}$. 
Proof. The reasoning is similar as in the case of Lemma 7 and Theorem 7 , but we consider $\operatorname{Mod}(p \mathrm{U} q)=\operatorname{Mod}(\mathrm{G}(\mathrm{F} r \rightarrow r) \wedge \mathrm{F}(q \wedge r) \wedge \mathrm{G}((r \wedge \neg q) \rightarrow p))\lceil\{p, q\}$ and $\operatorname{Mod}(\neg p \mathrm{U} q)=\operatorname{Mod}(\mathrm{F} q \rightarrow(\mathrm{F}(\neg p \wedge r) \wedge \mathrm{G}(\mathrm{Fr} \rightarrow \neg q))) \uparrow\{p, q\}$.

Theorem 10. $\mu \mathrm{TL}(\mathrm{U})$ is the interpolation closure of $\mathrm{PLTL}(\mathrm{U})$ over $\mathbf{T}_{\omega}$.

Proof. Let $\sigma$ be a modal signature. Now let $K \subseteq \operatorname{Str}_{\mathbf{T}_{\omega}}[\sigma]$ be definable by a $\mu \mathrm{TL}(\mathrm{U})$-sentence $\phi$ in signature $\sigma$. As $\mu \mathrm{TL}(\mathrm{U})$ is closed under negation, there is a $\mu \mathrm{TL}(\mathrm{U})$-sentence $\neg \phi$ in signature $\sigma$, which defines the complement $\bar{K} \subseteq \operatorname{Str}_{\mathbf{T}_{\omega}}[\sigma]$ of $K$ over $\operatorname{Str}_{\mathbf{T}_{\omega}}[\sigma]$. By Theorem 3, both $K$ and $\bar{K}$ are harmonious projective classes of PLTL(U). Now consider a temporal language $\mathcal{L} \supseteq \operatorname{PLTL}(U)$ with Craig interpolation over T. By Lemma $6, K$ and $\bar{K}$ are also harmonious projective classes of $\mathcal{L}$. By Proposition $3, \mathcal{L}$ is harmonious and by Lemma 7 , it follows that $K$ is definable in $\mathcal{L}$, i.e., $\mathcal{L} \supseteq \mu \mathrm{TL}(\mathrm{U})$.

\section{$6 \quad$ Finite Linear Orders}

We restricted our attention to the frame class $\mathbf{T}_{\omega}$, but our results easily extend to finite linear orders. Let $\mathbf{T}_{\text {fin }}$ be the class of frames $(D,<)$ where $D$ is a finite set and $<$ is a strict linear order on $D$. All the definitions and results that we gave relative to $\mathbf{T}_{\omega}$ also apply to $\mathbf{T}_{f i n}$. An analogous of Theorem 1 for $\mathbf{T}_{\text {fin }}$ can be obtained by considering automata on finite words. The proof of Proposition 2 can similarly be adapted by considering stutter-invariant automata on finite words. In the proof of Lemma 3, we can define $(\mathrm{X} \psi)^{*}$ as $\bigvee_{g \neq g^{\prime}}\left(\beta_{g} \cup\left(\beta_{g^{\prime}} \wedge \psi^{*}\right)\right)$ (i.e., we keep only the second disjoint, as no finite stutter free linear order exhibits two successor points satisfying the same set of proposition letters). The remaining of our arguments do not need any further adjustment.

\section{Conclusions and Future Work}

In this paper, we studied the temporal fragments of linear time $\mu$-calculus satisfying Craig interpolation, showing essentially that there are only three distinct such fragments: $\mu \mathrm{TL}$ itself, $\mu \mathrm{TL}(\mathrm{U})$, and $\operatorname{PLTL}(\mathrm{X})$. These results reconfirm the robustness of (linear time) $\mu$-calculus as compared to less expressive temporal logics. We are currently working on extending our results to other flows of time such as finite trees, infinite trees, and infinite linear orders other than the natural numbers (as in [5]). There are some important differences in these settings. For example, it is known (see [2]) that the branching time temporal logic with only Since and Until has Craig interpolation, while linear time fails to have this property. Also there is still no definitive consensus on the appropriate notion of stuttering for infinite branching time (see [13]).

\section{References}

[1] Eyal Amir and Sheila A. McIlraith. Partition-based logical reasoning for first-order and propositional theories. Artif. Intell., 162(1-2):49-88, 2005. 
[2] Carlos Areces and Maarten de Rijke. Interpolation and bisimulation in temporal logic. In Ruy J. Guerra B. de Queiroz and Marcelo Finger, editors, Proceedings of WoLLIC'98, pages 15-21, 1998.

[3] André Arnold and Damian Niwinski. Rudiments of $\mu$-Calculus, volume 146 of Studies in Logic and Foundations of Mathematics. North-Holland, 2001.

[4] John Barwise and Solomon Feferman. Model-theoretic logics. Springer, New York, 1985.

[5] Véronique Bruyère and Olivier Carton. Automata on linear orderings. $J$. Comput. System Sci., 73(1):1-24, 2007.

[6] Balder ten Cate. Model Theory for Extended Modal Languages. PhD thesis, University of Amsterdam, 2005. ILLC Dissertation Series DS-2005-01.

[7] William Craig. Three Uses of the Herbrand-Gentzen Theorem in Relating Model Theory and Proof Theory. Journal of Symbolic Logic, 22(3):269-285, 1957.

[8] Giovanna D'Agostino. Interpolation in non-classical logics. Synthese, 164(3):421-435, 2008.

[9] Giovanna D'Agostino and Marco Hollenberg. Logical Questions Concerning the $\mu$-Calculus: Interpolation, Lyndon and Lös-Tarski. Journal of Symbolic Logic, 65(1):310-332, 2000.

[10] Gerard R. Renardel de Lavalette. Interpolation in computing science: the semantics of modularization. Synthese, 164(3):437-450, 2008.

[11] E. Allen Emerson. Temporal and modal logic. In Jan van Leeuwen, editor, Handbook of Theoretical Computer Science, volume B, pages 995-1072. Elsevier, 1990.

[12] Kousha Etessami. Stutter-Invariant Languages, omega-Automata, and Temporal Logic. In Nicolas Halbwachs and Doron Peled, editors, Proceedings of $C A V$, pages 236-248, London, UK, 1999. Springer-Verlag.

[13] Ron Gross. Invariance under stuttering in branching-time temporal logic. Master's thesis, Israel Institute of Technology, Haifa, 2008.

[14] Roope Kaivola. Using Automata to Characterise Fixed Point Temporal Logics. PhD thesis, University of Edinburgh, 1997.

[15] Hans Kamp. Tense Logic and the Theory of Linear Order. PhD thesis, UCLA, Los Angeles, 1968.

[16] Leslie Lamport. What Good is Temporal Logic? In R. E. A. Mason, editor, Proceedings of the IFIP 9th World Computer Congress, pages 657-668. North-Holland/IFIP, 1983.

[17] Larissa Maksimova. Temporal logics with "the next" operator do not have interpolation or the Beth property. Siberian Mathematical Journal, 32(6):989993, 1991.

[18] Damian Niwinski. Fixed Points vs. Infinite Generation. In Proceedings of LICS, pages 402-409, 1988.

[19] Doron Peled and Thomas Wilke. Stutter-invariant temporal properties are expressible without the next-time operator. Inf. Process. Lett., 63(5):243246, 1997.

[20] Wolfgang Thomas. Handbook of formal languages, vol. 3: beyond words, chapter Languages, automata, and logic, pages 389-455. Springer-Verlag, New York, NY, USA, 1997. 